\title{
Effect of spatial distribution of fibers on elastic properties of unidirectional carbon/carbon composites
}

\author{
Mohan M.V.N ${ }^{12 *}$, Ramesh Bhagat Atul ${ }^{1}$ and Vijay Kumar Dwivedi ${ }^{2}$ \\ ${ }^{1}$ Scientist, Advanced Systems Laboratory, DRDO, Government of India, Hyderabad, India-500058. \\ ${ }^{2}$ Department of Mechanical Engg., IET, GLA University, Mathura, U.P., India.
}

\begin{abstract}
Carbon/Carbon composites finds its applications in several high temperature applications in the field of Space, Aviation etc. Designing of components or sub systems with carbon/carbon composites is a challenging task. It requires prediction of elastic properties with a very high accuracy. The prediction can be normally done by analytical, numerical or experimental methods. At the design stage the designers resort to numerical predictions as the experimental methods are not feasible during design stage. Analytical methods are complex and difficult to implement. The designers use numerical methods for prediction of elastic properties using Finite Element Modeling (FEM). The spatial distribution of fibers in matrix has an effect on results of prediction of elastic constants. The generation of random spatial distribution of fibers in representative volume element (RVE) challenging. The present work is aimed at study of effect of spatial distribution of fiber in numerical prediction of elastic properties of unidirectional carbon/carbon composites. MATLAB algorithm is used to generate the spatial distribution of fibers in unidirectional carbon/carbon composites. The RVE elements with various random fiber distributions are modeled using numerical Finite element Model using ABAQUS with EasyPBC plugin. The predicted elastic properties have shown significant variation to uniformly distributed fibers.
\end{abstract}

Keywords Spatial distribution of fibers, Unidirectional Carbon/Carbon Composites, Finite element modeling, elastic properties

\section{Introduction}

Carbon/Carbon composites finds its applications in several high temperature applications in the field of Space, Aviation; Air craft brakes, discs etc. Designing of components or sub systems with carbon/carbon composites is a challenging task. It requires prediction of elastic properties with a very high accuracy. The prediction can be normally done by analytical, numerical or experimental methods. At the design stage the designers resort to numerical predictions as the experimental methods are not feasible. Analytical methods are complex and difficult to implement. The designers use numerical methods for prediction of elastic properties using Finite Element Modeling (FEM). The spatial distribution of fibers in matrix has an effect on elastic constants. The generation of random spatial distribution of fibers in representative volume element (RVE) is challenging. The present work is aimed at study of effect of spatial distribution of fiber on elastic properties of unidirectional carbon/carbon composites. MATLAB algorithm is used to generate the spatial distribution of fibers in unidirectional carbon/carbon composites.

Aram bahmani et al. [1] assessed the randomly distributed representative volume elements employing a random event-driven molecular dynamics simulation. Chao Chang et al. [2] evaluated transverse effective mechanical properties of unidirectional composites with random distribution of fibers and interphase thickness. Jae Hyuk Lim et al. [3] proposed numerical scheme to evaluate the elasticproperties of fibers through inverse analysis with quasi-analytical gradients. Using this numerical scheme the authors evaluated elastic properties of T650-35fiberinaT65035/PMR-15 lamina using representative volume elements with random spatial distribution of fibers. Zhao Liu et al. [4] utilized Particle Swarm Optimization algorithm to generate random fiber distributions for fiber-reinforced composites. K. P. Babu et al. [5] used random sequential adsorption technique to generate RVE for prediction of the effective material properties. Juling Liu et al. [6] predicted transverse permeability through micro flow analysis of the RVE generated through an algorithm that can create microstructures of randomly distributed fiber with high-volume fraction. Taotao Zhang etal. [7] Presented a method for generation of random fiber distribution of fiber reinforced composites in transverse cross-section with high fiber volume

*Corresponding author: mvnmohan33@gmail.com 
fraction. The authors generated representative volume element by combining the proposed method with random disturbance algorithm and perfect elastic collision algorithm. Geng Li et al. [8] developed an algorithm event-driven molecular dynamics theory to develop representative volume element with random distribution of fibers in composites. Zhenqing Wang et al. [9] presented an algorithm for generation of representative volume element for long fiber reinforced composites with random distribution of fibers with high volume fraction. The authors included two procedures in the algorithm namely global crisscrossing and local disturbing. Shin-Mu Park et al. [10] used random fiber removal technique to generate random fiber distribution with diverse volume fractions. Rao et al. [11] analysed representative volume elements for different fiber architectures of carbon/carbon composites through finite element method. Yaser Ismail et al. [12] used discrete element method for random distribution of fibers in representative volume element. The authors chosen Eglass/MY750/HY917/DY063 that consists of Eglass fibre and epoxy resin. The numerical results have reasonable agreement with experimental results. Sun J et al. [13] presented a method to enhance strength and toughness using heat-treated interface modification for carbon/carbon composites. Lei Yang et al. [14] presented a method based on random sequential expansion for generation of random fiber distribution of fibers in fiber reinforced composites. Hai Qing et al. [15] performed two dimensional finite element simulations in metal matrix composites using silicon carbide reinforcement in aluminum alloy for evaluation of damage and deformation. WeijianGe et al. [16] the proposed a method for spatial random distribution of fibers based on iterative improvements with high volume fraction. Chao et al. [17] performed Micromechanical modeling of unidirectional composites. The authors have performed modelling with random fiber and interphase thickness distributions.Bo Cheng Jin et al. [18] has numerically created representative volume elements of unidirectional random filaments and fibers using PYTHON.The author has used modified random sequential adsorption for increasing the volume fraction of fibers. Venkata et al. [19] have compared the values of elastic constants evaluated by using analytical method and numerical methods. Some authors have studied analytical methods for evaluation of elastic properties [20].

In the present work the Effect of spatial distribution of fibers on numerical prediction of elastic properties of Unidirectional Carbon/Carbon composites is evaluated. The elastic properties are evaluated through numerical homogenization methods with uniform and random distribution of fibers. The random distribution is repeated for 10 iterations. The elastic properties evaluated using uniform distribution of fibers are compared with that of random distribution of fibers.

\section{Objective}

The objective of the current study is to study the effect of spatial distribution of fibers in unidirectional Carbon/Carbon composites on prediction of elastic properties. The steps involved are
- Create the iterations with uniform distribution and random fibers distribution using MATLAB [21] code

- Create Representative volume elements (RVE) for uniform and random distributions using ABAQUSCAE

- Perform post processing to evaluate elastic properties of carbon/carbon composites FEM using EasyPBC plugin [22].

- Compare elastic properties of from FEM using models of uniform distribution and random distribution.

\section{Elastic properties of unidirectional carbon/carbon composites}

Unidirectional carbon/carbon composites consist of carbon fiber in carbon matrix. The unidirectional carbon/carbon composites are normally assumed transversely isotropic materials. These materials contain two phases. The carbon fiber is called as reinforcement phase and it is assumed to be orthotropic material. The carbon matrix, the matrix phaseis assumed to be isotropic. The unidirectional carbon/carbon a transversely isotropic material is has five independent engineering constants. The engineering constants are shown in table 1 . They are longitudinal modulus $\left(E_{11}\right)$, transverse modulus $\left(\mathrm{E}_{22}\right)$, longitudinal shear modulus $\left(\mathrm{G}_{12}\right)$, transverse shear modulus $\left(\mathrm{G}_{23}\right)$ and major Poisson's ratio $\left(v_{12}\right)$. The minor Poisson's ratio $\left(v_{23}\right)$ is function of $E_{22}$ and $\mathrm{G}_{12}$. The elastic properties of the composite are evaluated using mechanical properties of fibers and matrix

Table 1. Engineering constants of unidirectional carbon/carbon composites

\begin{tabular}{|c|c|}
\hline Engineering Constant & Symbol \\
\hline Longitudinal modulus & $\mathrm{E}_{11}$ \\
\hline Transverse modulus & $\mathrm{E}_{22}$ \\
\hline Longitudinal shear modulus & $\mathrm{G}_{12}$ \\
\hline Transverse shear modulus & $\mathrm{G}_{23}$ \\
\hline Major Poisson's ratio & $v_{12}$ \\
\hline
\end{tabular}

\section{Numerical modelling of carbon/carbon composites}

\subsection{The numerical modelling include the following}

- Develop of iterations of uniform and random distribution of fibers with MATLAB algorithm

- Geometric modelling and pre-processing using ABAQUS CAE.

- Post processing to evaluate elastic properties using ABAQUS CAE with EasyPBC plugin.

\subsection{Development of iteration through MATLAB algorithm}

A MATLAB algorithm was developed to generate the iteration with random distribution of fibers. The developed code required inputs such as radius of the fiber, side length of the RVE and volume fraction. The code developed performs its intended function in three 
steps. In first step it creates a hard model with uniform distribution of fibers. The second step includes the random removal of fibers to achieve the desired the volume fraction of the fibers. The third step is stirring of the fibers. The MATLAB algorithm output include various iteration of random fiber distributions. The present study was done with fiber radius of $0.5 \mathrm{~mm}, \mathrm{RVE}$ side length $5 \mathrm{~mm}$ and with a volume fraction of 0.5 . Ten iterations obtained using MATLAB algorithm were shown in Fig. 1 to Fig. 10.

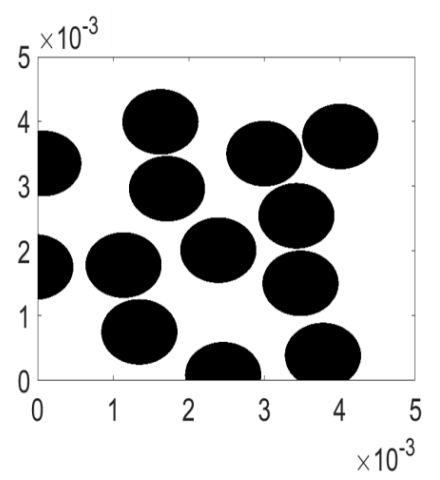

Fig. 1. Iteration 01

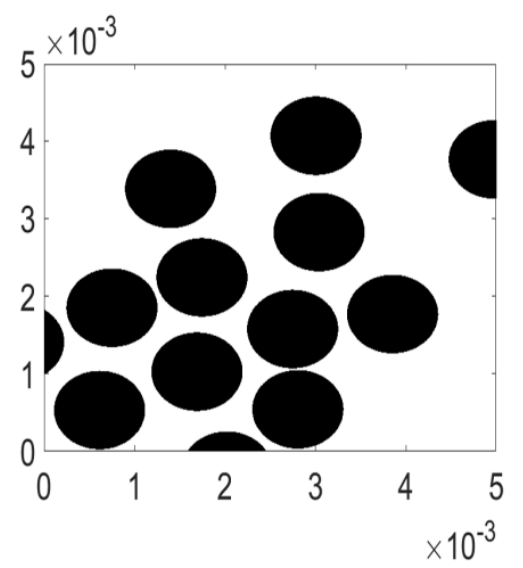

Fig. 3. Iteration 03

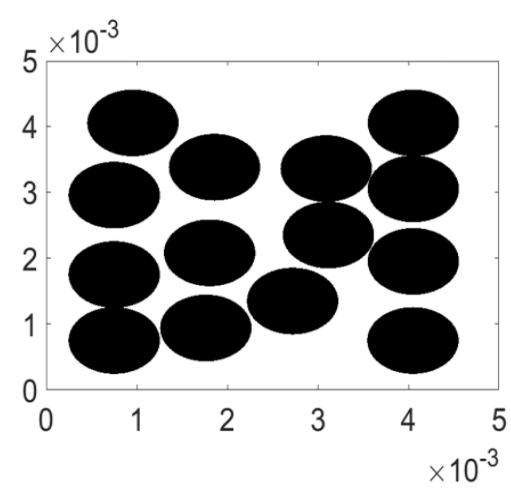

Fig. 5. Iteration 05

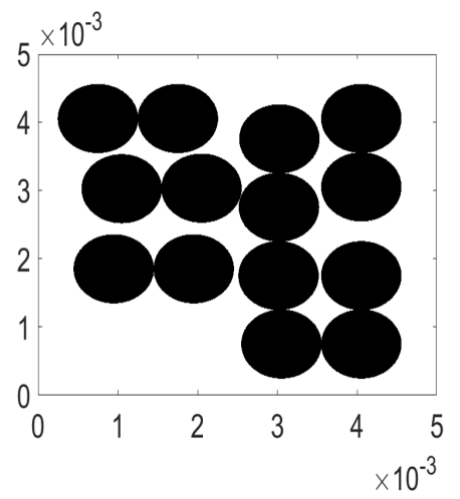

Fig. 2. Iteration 02

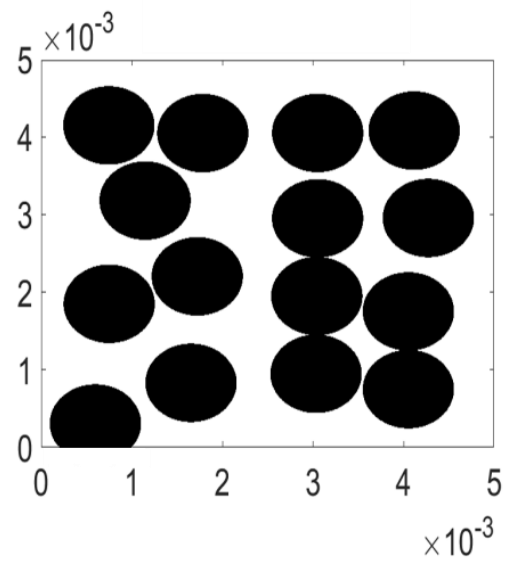

Fig. 4. Iteration 04

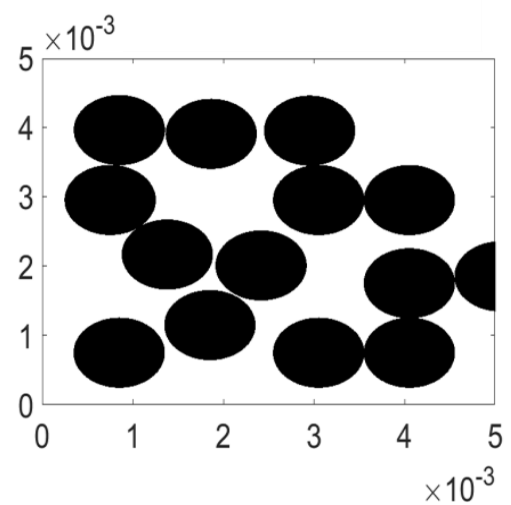

Fig. 6. Iteration 06 


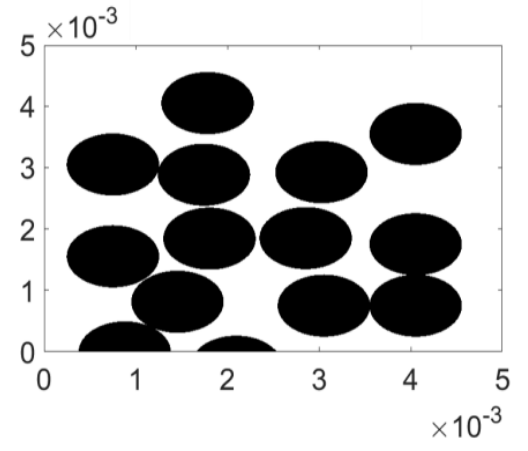

Fig. 7. Iteration 07

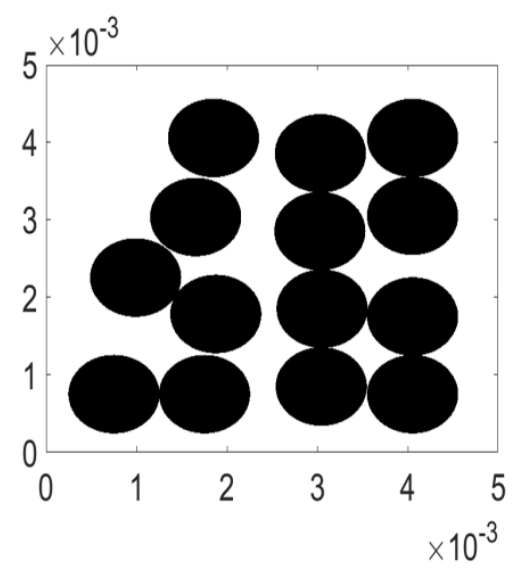

Fig. 9. Iteration 09

\subsection{Modelling and Meshing}

The output of MATLAB code is used to create geometric model in ABAQUSCAE. The representative volume elements are created with uniform and random distribution of fibers. The carbon fibers are considered

Table 2. Carbon Fiber Properties

\begin{tabular}{|c|c|c|}
\hline SI No & Property & Value \\
\hline 1 & $\mathrm{E}_{11}{ }^{\mathrm{f}}$ & $263.7 \mathrm{GPa}$ \\
\hline 2 & $\mathrm{E}_{22}{ }^{\mathrm{f}}$ and $\mathrm{E}_{33}{ }^{\mathrm{f}}$ & $19 \mathrm{GPa}$ \\
\hline 3 & $\mathrm{G}_{12}{ }^{\mathrm{f}}$ & $26.7 \mathrm{GPa}$ \\
\hline 4 & $\mathrm{G}_{13}{ }^{\mathrm{f}}$ & $26.7 \mathrm{GPa}$ \\
\hline 5 & $\mathrm{G}_{23}{ }^{\mathrm{f}}$ & $6.98 \mathrm{GPa}$ \\
\hline 6 & $v_{12}{ }^{\mathrm{f}}$ & 0.2 \\
\hline 7 & $v_{13}{ }^{\mathrm{f}}$ & 0.2 \\
\hline 8 & $v_{23}{ }^{\mathrm{f}}$ & 0.35 \\
\hline
\end{tabular}

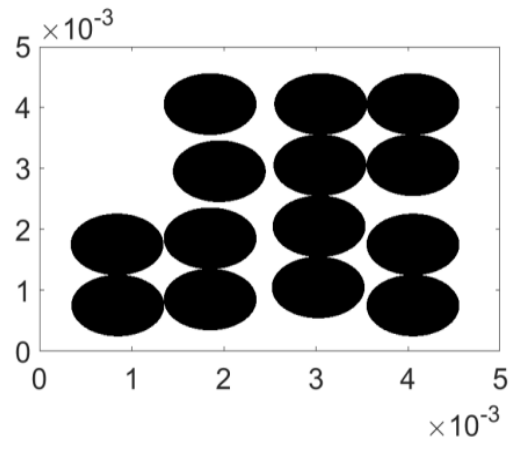

Fig. 8. Iteration 08

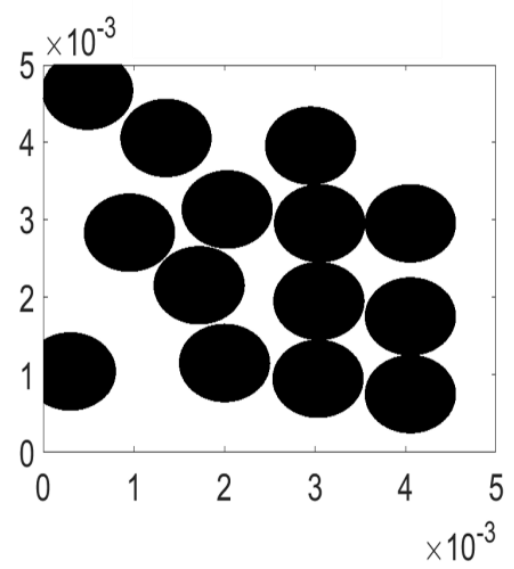

Fig. 10. Iteration 10

orthotropic and carbon matrix is considered isotropic. The properties carbon matrix and carbon fiber [11] for the material were assigned. The properties are given in table 2 and table 3.

Table 3. Carbon Matrix Properties

\begin{tabular}{|c|c|c|}
\hline SI No & Property & Value \\
\hline 1 & $\mathrm{Em}$ & $19 \mathrm{GPa}$ \\
\hline 2 & $v \mathrm{~m}$ & 0.2 \\
\hline 3 & $\mathrm{Gm}$ & $7.92 \mathrm{GPa}$ \\
\hline
\end{tabular}

The RVEs with uniform and random distribution of fibers is given in Fig.11 and Fig. 12. 


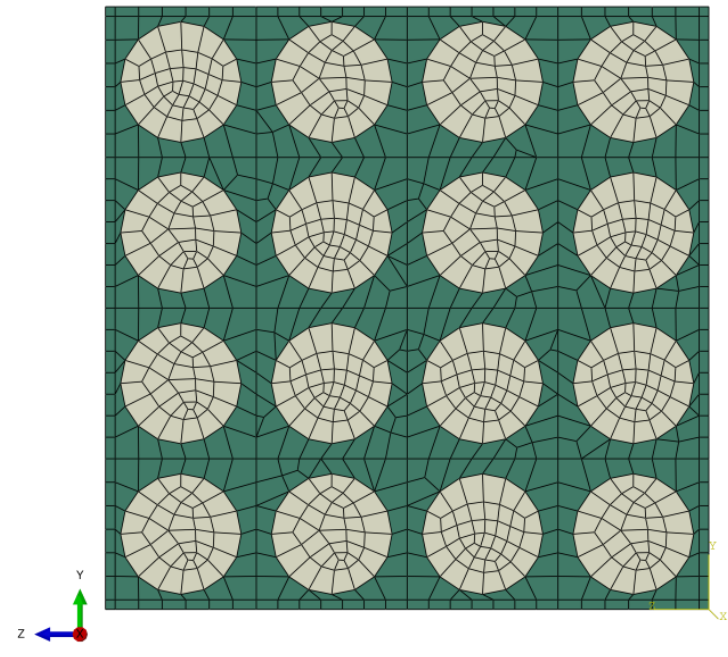

Fig. 11. Mesh generated on RVE with uniform distribution of fiber

\subsection{FEM analysis through ABAQUS CAE}

The model is then exported to ABAQUS for FEM study to evaluate of elastic properties. The representative volume element is treated a part of periodic material. The RVE homogenization needs uniform strains on for evaluation of elastic properties. Periodic boundary conditions ensure that the deformed surfaces of RVE remain periodic. The periodic boundary conditions are possible to be implemented in Abaqus with EasyPBC

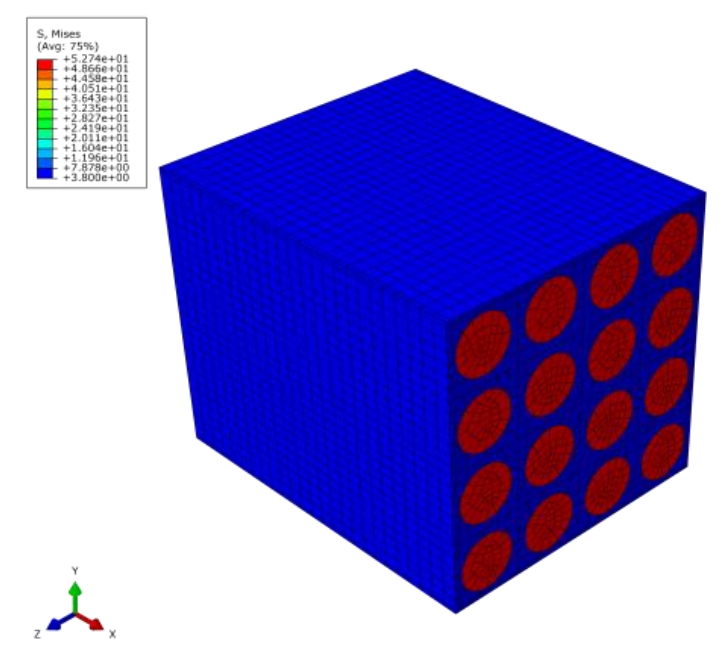

Fig. 13. Stress developed on the body while calculating $\mathrm{E}_{11}$ with uniform distribution of fiber.

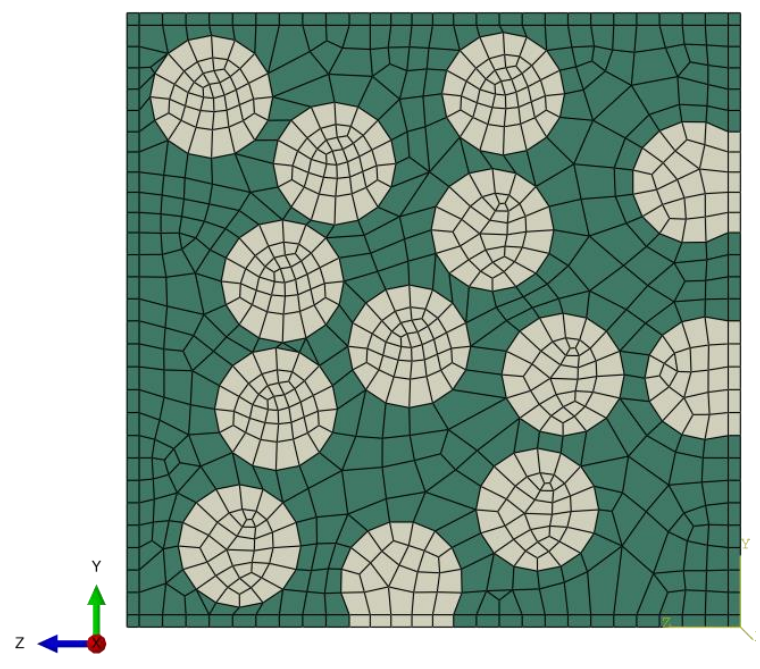

Fig. 12. Mesh generated on RVE with random distribution of fiber

plugin. In the present study the elastic properties are evaluated using EasyPBC plugin. The stresses at the boundary conditions for material with uniformly distributed fibers is shown in Fig.13 to Fig. 20.

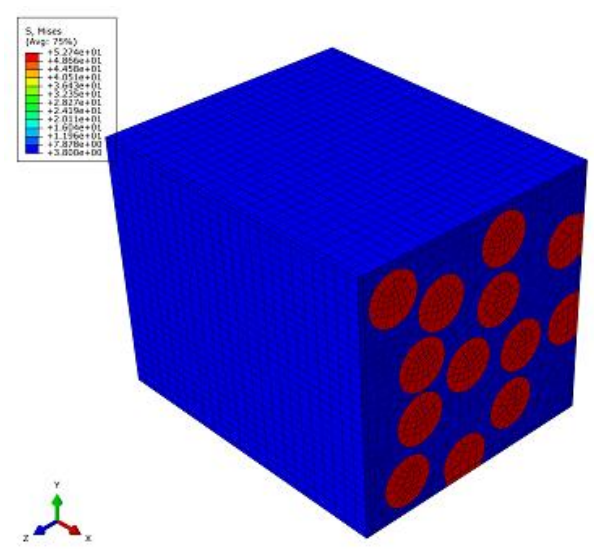

Fig. 14. Stress developed on the body while calculating $\mathrm{E}_{11}$ with random distribution of fiber. 


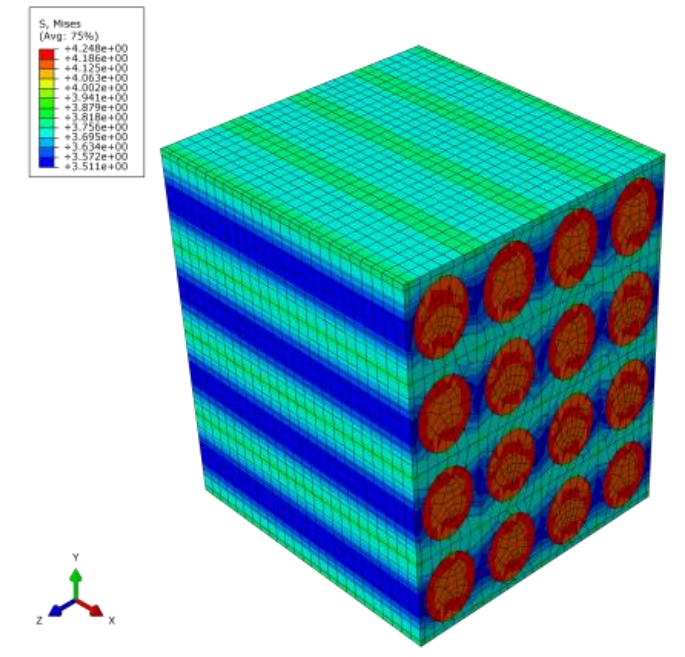

Fig. 15 Stress developed on the body while calculating $\mathrm{E}_{22}$ with uniform distribution of fiber.

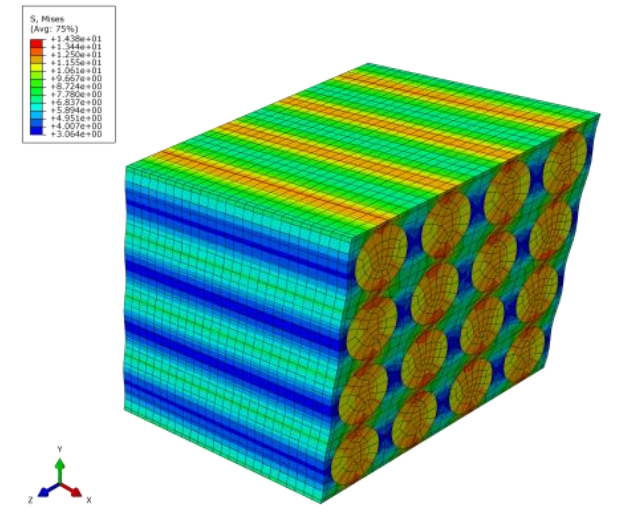

Fig. 17 Stress developed on the body while calculating $\mathrm{G}_{12}$ with uniform distribution of fiber.

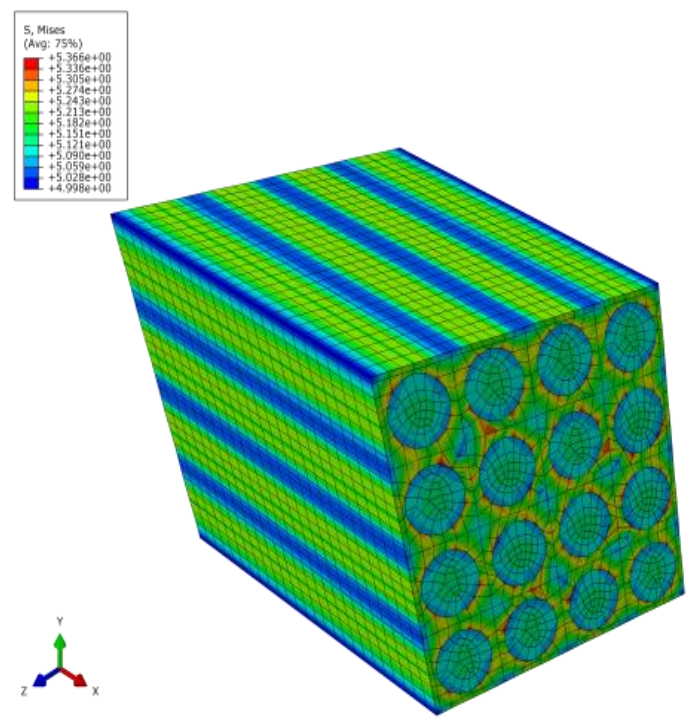

Fig. 19 Stress developed on the body while calculating G23 with uniform distribution of fiber.

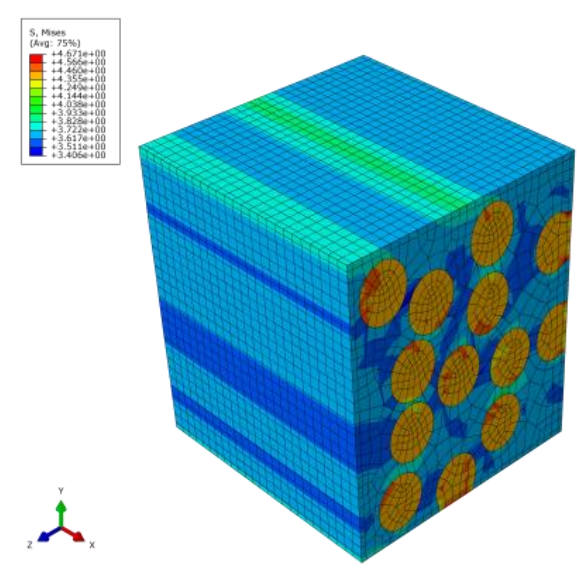

Fig. 16 Stress developed on the body while calculating $\mathrm{E}_{22}$ with random distribution of fiber.

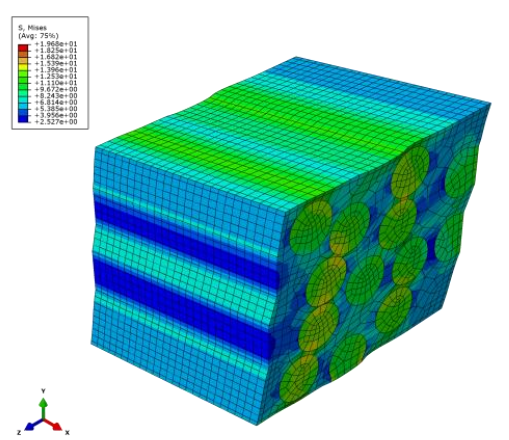

Fig. 18 Stress developed on the body while calculating $\mathrm{G}_{12}$ with random distribution of fiber.

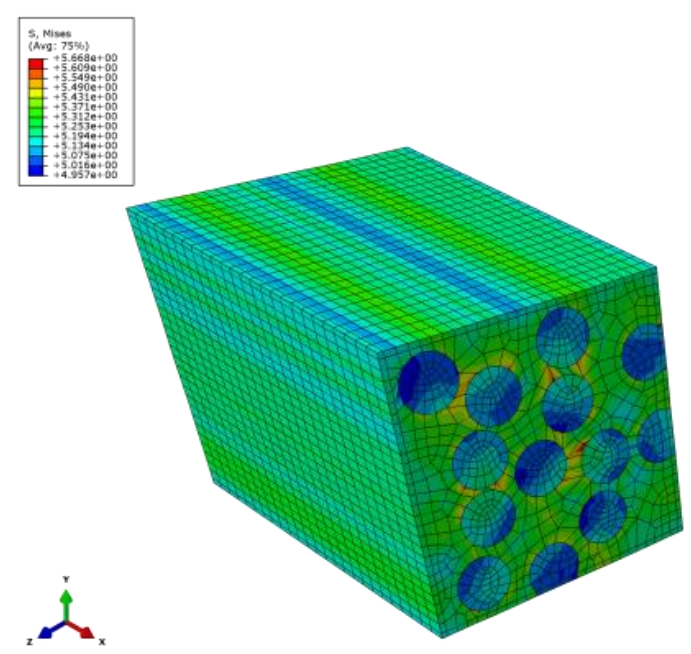


Fig. 20 Stress developed on the body while calculating $\mathrm{G}_{23}$ with random distribution of fiber

Table 5 Comparison elastic prperties

\section{RESULTS AND DISCUSSION}

The effect of spatial distribution of fibers is on elastic constants is evaluated through numerical methods. The Table 4 shows the elastic properties evaluated through various iterations of fiber distribution. Table 5 shows the variation of elastic properties between the random and uniform distributions of UD c/c composite.

Table 4. Elastic properties

\begin{tabular}{|c|c|c|c|c|c|}
\hline $\begin{array}{c}\text { Iterati } \\
\text { ons }\end{array}$ & $\begin{array}{c}\text { Longitu } \\
\text { dinal } \\
\text { Modulu } \\
\text { s (E } \text { E }_{11} \text { in } \\
\text { GPa) }\end{array}$ & $\begin{array}{l}\text { Trans } \\
\text { verse } \\
\text { Modul } \\
\text { us ( } \text { E } 22 \\
\text { in } \\
\text { GPa) }\end{array}$ & $\begin{array}{c}\text { Longitu } \\
\text { dinal } \\
\text { shear } \\
\text { Modulu } \\
\text { s }\left(\mathbf{G}_{12}\right. \\
\text { in } \mathbf{G P a})\end{array}$ & $\begin{array}{c}\text { Trans } \\
\text { verse } \\
\text { shear } \\
\text { modul } \\
\text { us } \\
\left(G_{23} \text { in }\right. \\
\text { GPa) }\end{array}$ & $\begin{array}{c}\text { Ma } \\
\text { jor } \\
\text { pois } \\
\text { on } \\
\text { rati } \\
\text { o } \\
\text { v12 } \\
\end{array}$ \\
\hline 1 & 114.42 & 19.39 & 12.54 & 7.53 & 0.2 \\
\hline 2 & 118.57 & 19.39 & 12.67 & 7.51 & 0.2 \\
\hline 3 & 115.83 & 19.39 & 12.35 & 7.53 & 0.2 \\
\hline 4 & 130.87 & 19.36 & 13.33 & 7.47 & 0.2 \\
\hline 5 & 117.15 & 19.39 & 12.45 & 7.52 & 0.2 \\
\hline 6 & 116.34 & 19.39 & 12.54 & 7.52 & 0.2 \\
\hline 7 & 123.1 & 19.37 & 13.01 & 7.51 & 0.2 \\
\hline 8 & 118.79 & 19.39 & 12.48 & 7.51 & 0.2 \\
\hline 9 & 116.87 & 19.39 & 12.36 & 7.52 & 0.2 \\
\hline 10 & 128.2 & 19.37 & 13.18 & 7.48 & 0.2 \\
\hline $\begin{array}{c}\text { Unifor } \\
\mathrm{m} \\
\text { Distrib } \\
\text { ution }\end{array}$ & 138.86 & 19.35 & 13.76 & 7.43 & 0.2 \\
\hline
\end{tabular}

The elastic properties of the unidirectional carbon/carbon composites are predicted using numerical homogenization methods for uniform and random distribution of fibers. The comparison of the predicted properties are concluded below.

- There is a significant variation in in the evaluated values of elastic modulus in longitudinal direction. However the transverse young modulus has good agreement between uniform and random distributions.

- The shear modulus in longitudinal direction show a variation of $8.4 \%$ between the values obtained for Uniform and random distributions. The values of shear modulus in transverse direction has a good agreement between uniform and random distributions.

- There is no variation in estimated values of major poisons ratio.

\section{CONCLUSIONS AND FUTURE}

[1] Aram Bahmani, Geng Li, Thomas L. Willett, John Montesano (2018) Three-dimensional microscopic assessment of randomly distributed representative volume elements for high fiber volume fraction unidirectional composites, Composite Structures 192 (2018) 83 153-164.

[2] Chao Chang, Yuxuan Zhang, HuiWang(2019) Micromechanical modeling of unidirectional

\begin{tabular}{|c|c|c|c|}
\hline $\begin{array}{c}\text { Elastic } \\
\text { Property }\end{array}$ & $\begin{array}{c}\text { Uniform } \\
\text { Distribution } \\
\text { (GPa) }\end{array}$ & $\begin{array}{c}\text { Random } \\
\text { Distribution } \\
\text { of fibers } \\
\text { (GPa) }\end{array}$ & $\begin{array}{c}\text { \% } \\
\text { Difference }\end{array}$ \\
\hline $\mathrm{E}_{11}$ & 138.86 & 120.01 & -15.7 \\
\hline $\mathrm{E}_{22}$ & 19.35 & 19.38 & 0.154798762 \\
\hline $\mathrm{G}_{12}$ & 13.76 & 12.69 & -8.43183609 \\
\hline $\mathrm{G}_{23}$ & 7.43 & 7.51 & 1.06 \\
\hline $\mathrm{v}_{12}$ & 0.2 & 0.2 & 0 \\
\hline
\end{tabular}

composites with random fiber and interphase thickness distributions, Arch ApplMechhttps://doi.org/10.1007/s00419-01901595-0

[3] Jae Hyuk Lim, Milan Henry, Do-Soon Hwang, Dongwoo Sohn (2016) Numerical prediction of fiber mechanical properties considering random microstructures using inverse analysis with quasianalytical gradients, Applied Mathematics and Computation 273 (2016) 201-216

[4] Zhao Liu, Chao Zhu, Ping Zhu (2015) Generation of Random Fiber Distributions for Unidirectional Fiber-Reinforced Composites Based on Particle Swarm Optimizer, 5(3C): 162-168. DOI: 10.5923/c.materials.201502.32

[5] K.P. Babu, P.M. Mohite, C.S. Upadhyay, Development of an RVE andits stiffness predictions based on mathematical homogenization theory for short fibre composites, International Journal of 
Solids and Structures (2017), doi: 10.1016/j.ijsolstr.2017.10.011

[6] Junling Liu, Li Chen, Junbo Xie, Ziyuan Lin (2020) Micro-flow model with a new algorithm of random fiber distribution over the transverse cross-section, Polymer composites. 2020; 1-10.

[7] Taotao Zhang, Ying Yan (2015) A Comparison between Random Model and Periodic Model for Fiber-Reinforced Composites Based on a New Method for Generating Fiber Distributions, Polymer composites-2015 DOI 10.1002/pc

[8] Geng Li, Farzad Sharifpour, Aram Bahmani, John Montesano, A new approach to rapidly generate random periodic representative volume elements for microstructural assessment of high volume fraction composites.J.04.031

[9] Zhenqing Wang, Xiaoqiang Wang, Jifeng Zhang, Wenyan Liang, Limin Zhou (2011) Automatic generation of random distribution of fibers in longfiber-reinforced composites and meso mechanical simulation, Materials and Design 32 (2011) 885891

[10] Shin-Mu Park, Jae Hyuk Lim, Myeong Ryun Seong, Dongwoo Sohn(2019) Efficient generator of random fiber distribution with diverse volume fractions by random fiber removal, Composites Part B 167 (2019) 302-316

[11] M. Venkat Rao, P. Mahajan, R.K. Mittal (2008) Effect of architecture on mechanical properties of carbon/carbon composites, Composite Structures, 83 131-142.

[12] Yaser Ismail, Dongmin Yang, Jianqiao Ye (2016) discrete element method for generating random fibre distributions in micro mechanical models of fibre reinforced composite laminates, Composites Part B 90 (2016) 485-492

[13] Sun J, Li H, Han L, Song Q, Enhancing both strength and toughness of carbon/carbon composites by heat-treated interface modification, Journal of Materials Science and amp; Technology (2018), https://doi.org/10.1016/j.jmst.2018.09.055

[14] Lei Yang, Ying Yan, Zhiguo Ran, Yujia Liu (2013) A new method for generating random fibre distributions for fibre reinforced composites, Composites Science and Technology 76 (2013) $14-20$

[15] Hai Qing (2013) Automatic generation of 2D micromechanical finite element model of siliconcarbide/aluminum metal matrix composites: Effects of the boundary conditions, Materials and Design 44 (2013) 446-453
[16] Weijian Ge, Libo Wang, Yongle Sun, Xuqing Liu (2019) An efficient method to generate random distribution of fibers in continuous fiber reinforced composites. Polymer Composites. 2019;1-8.

[17] Chao, X., Qi, L., Cheng, J., Tian, W., Zhang, S., Li, H., Numerical evaluation of the effectof pores on effective elastic properties of Carbon/Carbon composites, Composite Structures (2018), doi: https://doi.org/10.1016/j.compstruct.2018.05.014

[18] Bo Cheng Jin, Assimina A. Pelegri(2011)ThreeDimensional Numerical Simulation of Random Fiber Composites With High Aspect Ratio and High Volume Fraction, Journal of Engineering materials and Technology October 2011, Vol. 133 / 041014-1

[19] Venkata Naga Mohan Manchiraju, Atul Bhagat\& Vijay Kumar Dwivedi (2021): Prediction of elastic properties of unidirectional carbon/carbon composites using analytical and numerical homogenization methods, Advances in Materials and Processing Technologies, DOI: 10.1080/2374068X.2021.1953922

[20] Autar K. Kaw (2006) Mechanics of Composite Materials, 2nd edition, Taylor \& Francis, CRC Press, ISBN 9780815351481.

[21] The Mathworks. MATLAB Simulink R2009a ${ }^{\circledR}$ Manual, 2009

[22] Omairey S., Dunning P., Sriramula S., (2018) Development of an ABAQUS Plugin Tool for Periodic RVE Homogenisation, Engineering with Computers, https://doi.org/10.1007/s00366-0180616-4 